Bol. Acad. peru. leng. 65. 2019 (151-165)

\title{
EL SUFIJO DE PLURAL EN CASTELLANO: FRECUENCIA Y COGNICIÓN
}

\author{
THE PLURAL SUFFIX IN SPANISH: \\ FREQUENCY AND COGNITION
}

\author{
Raymundo Casas Navarro \\ Universidad Nacional Mayor de San Marcos
}

\section{Resumen:}

Los estudios sobre frecuencia léxica parten de la denominada ley de Zipf (1949), según la cual las palabras más frecuentes en las lenguas tienden a la menor longitud (en términos de número de fonemas o sílabas), lo que revela una asimetría como una propiedad prevalente del lenguaje. Aunque tal regularidad estadística se suele analizar desde el expediente de la economía verbal en los gestos articulatorios, la perspectiva más reciente trata de dilucidar los aspectos relacionados con la memoria léxica o con la cognición en general. En este escenario, nos interesa abordar el fenómeno de la frecuencia reversible en los plurales: contra la propensión general de que las formas singulares sean más frecuentes que las formas plurales, hay un grupo exiguo de palabras en el que las formas plurales exhiben mayor frecuencia que las correspondientes formas singulares. Teniendo en cuenta la imponente base de datos conocida como el listado de frecuencias del Corpus de Referencia del Español Actual (CREA), hemos analizado una muestra de 40 palabras que ostenta la frecuencia reversible de manera consistente. Nuestro cometido 
es intentar formular una explicación plausible en el marco promisorio de la fonología cognitiva en términos de una relación entre el polo fonológico y el polo semántico, razón por la cual postulamos una relación conceptual entre el acceso al léxico y la frecuencia de palabras.

\section{Abstract:}

Studies on lexical frequency start from the so-called Zipf law (1949), according to which the most frequent words in languages tend to be the shortest in length (in terms of number of phonemes or syllables), which reveals an asymmetry as a prevalent property of language. Although such statistical regularity is usually analyzed from the records of verbal economy in articulatory gestures, the most recent perspective tries to elucidate the aspects related to lexical memory or cognition in general. In this context, we are interested in approaching the phenomenon of reversible frequency in plurals: while there is a general tendency for singular forms to be more frequent than plural forms, there is a small group of words in which plural forms exhibit greater frequency than the corresponding singular forms. Taking into account the overwhelming database known as the list of frequencies from the Corpus de Referencia del Español Actual (CREA) (Reference Corpus of Current Spanish), we have analyzed a sample of 40 words that flaunts the reversible frequency in a consistent manner. Our task is to try to formulate a plausible explanation in the promising framework of cognitive phonology in terms of a relationship between the phonological pole and the semantic pole, which is why we postulate a conceptual relationship between lexical access and word frequency.

Palabras clave: dominancia plural, frecuencia reversible, polo semántico.

Key words: plural dominance, reversible frequency, semantic pole.

Fecha de recepción:

Fecha de aceptación:
$30 / 03 / 2019$

$31 / 05 / 2019$ 


\section{Introducción}

Según la teoría lingüística, las computaciones sintácticas operan sobre las unidades léxicas de acuerdo con el modelo avanzado por Pinker: wordsand-rules (Pinker 1997). Vale decir, la facultad del lenguaje que consiste en la habilidad para producir y comprender los mensajes estriba en dos procesos: el acceso al léxico (words) y las computaciones (rules).

Como dilucida Levelt (2001), la producción léxica tiene una conspicua base semántica, y el acceso al léxico implica hacer referencia a un componente fundamental: el lexicón mental. En el habla normal, se producen de 2 a 4 palabras por segundo y la competencia sintáctica establece rutas de derivación que permiten la construcción de una cantidad imponente de mensajes.

Las formas singulares y plurales corresponden a la flexión. A diferencia de los procesos de derivación, la flexión no altera la categoría de la palabra: $\left[_{\mathrm{N}}\right.$ paloma $] \rightarrow\left[_{\mathrm{N}}\right.$ paloma $\left.+\mathrm{s}\right]$. En cambio, al agregarse un sufijo derivativo, puede alterarse la categoría: [V durar] $\rightarrow$ [Adj $\operatorname{dura}(\mathrm{r})+$ ble].

Analizando los sistemas flexivos, se determinan ciertas asimetrías que se dan en las lenguas (Haspelmath \& Sims, 2010:265). La asimetría entre el singular y plural se da en muchas lenguas, por ejemplo, en francés, latín y ruso (apud Haspelmath \& Sims, 2010):

\begin{tabular}{|c|c|c|}
\hline Lengua & Singular & Plural \\
\hline Francés & $74.3 \%$ & $25.7 \%$ \\
\hline Latín & $85.2 \%$ & $14.8 \%$ \\
\hline Ruso & $77.7 \%$ & $22.3 \%$ \\
\hline
\end{tabular}


La frecuencia tiene efectos en la memoria humana (Allen \& Garton 1968), de donde se puede derivar que una palabra con alta frecuencia ostenta un alto grado de saliencia desde la perspectiva de la ciencia cognitiva. Aunque la frecuencia léxica es un tópico de análisis de superficie (Köhler, Altmann \& Grzybeck 2009), puede conducirnos a mirar más adentro, por ejemplo, en el polo semántico. Así, la famosa ley de Zipf revela un aspecto superlativo de la cognición humana. Según la regularidad observada por Zipf (1949), la longitud de las formas léxicas se mide en términos del número de sílabas, es decir, las palabras cortas son las más frecuentes, dado que la longitud es una función de frecuencia. Todo indica que la ley de Zipf es una propensión universal en la medida en que se ha registrado en una buena cantidad de lenguas (Dehaene \& Mehler, 1992), y esto puede ser señal de mecanismos subyacentes que van más allá de la mera economía articulatoria. La propensión de Zipf recusa la noción de equiprobabilidad léxica (es decir, en una lengua cualquiera, cualquier palabra exhibe la misma frecuencia de ocurrencia). En el marco de la teoría cognitiva, la propensión de Zipf se debe analizar en términos de factores cognitivos como la memoria o en virtud de categorías asociadas al significado. Por ejemplo, en el habla cotidiana de los estudiantes de la PUCP, es más frecuente la expresión "Cato» que «Católica». Algo similar sucede con "profe» en vez de «profesor». Inclusive, la forma sincopada [pro'sor] 'profesor' es muy frecuente. La economía articulatoria es fundamental, pero también concurren factores ligados con la emoción o con el sentido de familiaridad.

Las frecuencias léxicas tienen siempre un significado y dependen mucho del universo del discurso. El contexto desempeña un rol en la frecuencia: en una biografía de Miguel Grau, el sustantivo Angamos tendrá más frecuencia de aparición que Motupe. Asimismo, muy probablemente, la palabra narratología tendrá una ocurrencia nula en un texto de física teórica, pero la terminación -logía puede presentar cierta frecuencia. Ello quiere decir que los sufijos tienen una frecuencia que trasciende la tipología textual.

En los estudios sobre la frecuencia léxica, el procesamiento del singular y del plural reviste cierta importancia (New, Segui, Ferrand 
\& Rastle, 2004). El singular /'gato/ es más frecuente que el plural /'gatos/, pero el plural /'labios/ es más frecuente que el singular /'labio/. En esta frecuencia reversible, hay una base semántica insoslayable, lo que quiere decir que el llamado polo fonológico está abierto al polo semántico, a la conceptualización sobre el mundo, sobre el cuerpo, sobre las experiencias vitales.

Tabla 1

\section{Palabras más frecuentes}

\begin{tabular}{|l|l|}
\hline Francés & Español \\
\hline 1. de & 1. de \\
2. le & 2. la \\
3. la & 3. que \\
4. et & 4. el \\
5. les & 5. en \\
6. des & 6. y \\
7. est & 7. a \\
8. un & 8. los \\
9. une & 9. se \\
10. du & 10. del \\
\hline (apud Haspelmath \& Sims 2010) & (apud CREA) \\
\hline
\end{tabular}

Aplicando la ley de Zipf, en las lenguas el patrón de dominancia singular es muy conspicuo. Según este patrón, las formas singulares de las palabras son más frecuentes que las respectivas formas plurales. Sin embargo, también se da el patrón de dominancia plural, en la medida en que las formas plurales de las palabras son más frecuentes que las respectivas formas singulares. Así, en dutch, la forma bruid 'novia' es más frecuente que la forma bruiden 'novias' de acuerdo con el patrón de dominancia singular, pero el dutch también exhibe el patrón de dominancia plural: la forma wolken 'nubes' es más frecuente 
que la forma wolk 'nube'. Como se ha probado en estudios en torno a la lengua dutch, hay efectos fuertes de la frecuencia en tareas de decisión léxica que implican un modelo de doble ruta, lo que implicaría que ciertas formas plurales exhiben prominencia cognitiva (Baayen, Dijkstra \& Schreuder, 1997). Lo que observamos en dutch se aplica en castellano, de tal manera que tenemos dos patrones de dominancia diferentes:

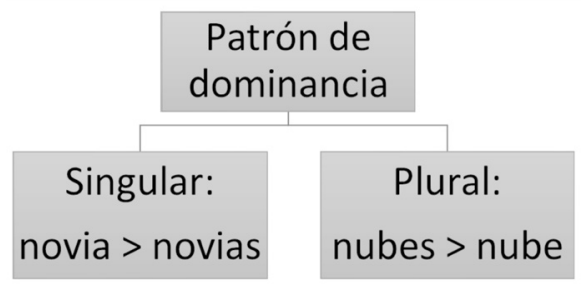

Aunque el patrón de dominancia singular se ajusta a la ley de Zipf (en castellano son más frecuentes las siguientes formas singulares: miedo, corbata, asunto, verano, espejo, miseria, etc.), existe el patrón de dominancia plural en algunos vocablos: labios, piernas, minutos, páginas, recursos, rodillas, sábanas, párpados, zapatos, etc. (Alameda \& Cuetos, 1995). Lo mismo sucede en la lengua inglesa, donde la palabra sum se ajusta al patrón de dominancia singular, pero la palabra eyes se ajusta al patrón de dominancia plural.

\section{Problema e hipótesis}

La frecuencia léxica es el aspecto más robusto en los análisis de reconocimiento léxico (Norris, 2006) y en el seno de la fonología cognitiva (Bybee, 1994). En el análisis de las formas plurales, hay que establecer el engarce con un factor cognitivo de dominancia: patrón de dominancia singular o patrón de dominancia plural. Es más, de acuerdo con Calude \& Pagel (2011), la frecuencia de uso es una fuerza superlativa, lo que se nota diáfanamente en que la célebre lista de Swadesh tiene una considerable correlación en muchas lenguas no emparentadas. 
El procesamiento del plural añade carga al procesamiento (Sereno \& Allard, 1997). El plural del inglés /dog/ implica añadir un fonema /s/. En castellano, sucede algo parecido, al agregarse un sufijo flexivo: $<$ perro $+-s>$. Los tiempos de reacción en plural suelen ser más lentos por una consideración de parsing. Lo anterior permite inferir la mayor frecuencia de las formas singulares; sin embargo, hay ciertos vocablos que se ajustan al patrón de dominancia plural, lo que implica un costo adicional en el procesamiento que debiera ser explicado (Domínguez, Cuetos \& Segui, 1999).

En consecuencia, nuestro problema de investigación se puede formular de la siguiente manera:

Dado que el patrón de dominancia plural implica añadir elementos en el polo fonológico $\{-s 0-e s\}$ y esto acarrea un costo articulatorio, ¿ ihay factores semántico-cognitivos propios del polo semántico que compensarían el mayor esfuerzo del polo fonológico?

Este problema presupone constatar la existencia de la frecuencia reversible (la dominancia plural en ciertos vocablos) y la necesidad de postular factores semántico-cognitivos en la medida en que la frecuencia reversible supone contrarrestar la ley de Zipf.

Para ensayar una respuesta posible para el problema de investigación formulado, postulamos la siguiente hipótesis:

El patrón de dominancia plural exbibe una frecuencia reversible que, sin embargo, no es un óbice para el acceso al léxico, dado que ciertos esquemas propios del polo semántico contrarrestan el mayor costo del polo fonologico. En efecto, en ciertos vocablos conspicuamente plurales, operan consideraciones semánticas asociadas con las ideas de inherencia plural, colectividad, intensidad sumatoria y experiencia plural corpórea.

Gracias a esta hipótesis, podemos dar cuenta de la frecuencia reversible propia de la dominancia plural, y dado que se trata de consideraciones generales con repercusiones interlingüísticas, determinamos que no es una 
hipótesis ad hoc. Esta hipótesis se enmarca dentro de las líneas maestras de la fonología cognitiva porque esgrime un argumento para sostener que hay engarces entre los polos fonológico y semántico. Asimismo, explica la frecuencia en términos de dominancia cognitiva.

\section{Metodología}

El análisis que hacemos parte de algunas restricciones metodológicas necesarias. En primer lugar, trabajamos con las palabras que forman plural con el sufijo $\{-\mathrm{s}\}$ o el alomorfo $\{-\mathrm{es}\}$, de manera que dejamos de lado las palabras cuyo plural recurre a otros modos de formación (verbigracia, 'yo' $\rightarrow$ 'nosotros'). En segundo lugar, excluimos las palabras denominadas singularia tantum (sed, tez, caos, etc.) y las palabras denominadas pluralia tantum (enseres, arras, nupcias, etc.). En tercer lugar, trabajamos solo con la categoría nominal, y alejamos del análisis palabras gramaticales como determinantes o cuantificadores.

El método que aplicamos tiene muchas imbricaciones con lo que se conoce como lingüística del corpus, es decir, una actividad rigurosa que consiste en trabajar con bases de datos potentes por el hecho de su calidad de procesamiento o por consideraciones de extensión cuantitativa. Felizmente, los especialistas en lingüística hispánica contamos con una serie de corpora procesada por el equipo técnico de la Real Academia Española y que está disponible de manera integral para llevar a cabo una serie de indagaciones sobre la sincronía o diacronía de la lengua castellana.

En consecuencia, para determinar el patrón de dominancia plural con un firme soporte de frecuencia, trabajamos con una imponente base de datos denominada «Listado de frecuencias», del Corpus de Referencia del Español Actual (CREA), una suma de documentos distribuidos por la Real Academia Española (www.rae.es). El listado se sustenta en un trabajo sobre 140 mil documentos (en torno a más de cien materias distintas) y dispone de 154 millones de formas. El material se construyó con diversos documentos producidos en el periodo de 1975 a 2004. Aunque es una base de datos que puede tener lagunas metodológicas, consideramos que supera con creces los estudios que se sustentan en 
una pequeña nómina de ejemplos y que, en consecuencia, nos lleva a generalizaciones precarias.

Sobre la base de las diez mil palabras más frecuentes del español («Listado de frecuencias» del CREA), determinaremos una muestra de 40 palabras con frecuencia reversible (i.e., las formas plurales son más frecuentes que las respectivas formas singulares).

En este trabajo, consideramos la frecuencia ordinal, puesto que el número de orden en el listado es un dato clave para calibrar la frecuencia de la palabra. Así, el patrón de dominancia plural, en términos operativos, implica que la forma plural tiene un número ordinal previo al que exhibe la forma singular. Luego, tratamos de perfilar una dilucidación concerniente al polo semántico para determinar la plausibilidad de nuestra hipótesis (ver 4.4).

\section{Resultados y discusión}

En el listado de frecuencias (CREA), las palabras con patrón de dominancia singular constituyen, indudablemente, la amplia mayoría, lo que revela una asimetría notable del lenguaje. Esta asimetría se interpreta de manera nítida en términos de la ley de Zipf, por cuanto la economía articulatoria predice que la forma singular («canción») será más frecuente que la forma plural («canciones»). En seguida, presentamos una pequeña muestra representativa, donde el orden de frecuencia revela mayor frecuencia, es decir, la forma «calle» tiene el orden 378 (su frecuencia normalizada es de 205.36) y la forma «calles» tiene el orden 1055 (su frecuencia normalizada es de 85.71).

\section{$\underline{\text { Patrón de dominancia singular (frecuencia ordinal) }}$}

\begin{tabular}{|l|l|}
\hline 1. (378) calle $\rightarrow$ (1055) calles & 6. (131) momento $\rightarrow$ (712) momentos \\
2. (90) día $\rightarrow(150)$ días & 7. (372) amor $\rightarrow(6128)$ amores \\
3. (101) mundo $\rightarrow$ (6572) mundos & 8. (442) arte $\rightarrow(2403)$ artes \\
4. (205) noche $\rightarrow$ (1782) noches & 9. (1637) sombra $\rightarrow(3281)$ sombras \\
5. (1158) esposa $\rightarrow$ (9651) esposas & 10. (1915) pecho $\rightarrow(6940)$ pechos \\
\hline
\end{tabular}


Aunque el patrón de dominancia singular es el más conspicuo en las lenguas y también en lengua castellana, lo que guarda consonancia con la ley de Zipf, en el listado de frecuencias también tenemos palabras con el patrón de dominancia plural. Vamos a trabajar con una muestra de 40 palabras que evidencian la dominancia plural, lo que se demuestra en el listado con la frecuencia ordinal: la prelación en el orden de frecuencia indica una mayor frecuencia. Así, la forma plural «ojos» ocupa el orden 263 (su frecuencia normalizada es de 277.73) y la forma «ojo» ocupa el orden 1821 (su frecuencia normalizada es de 52.50).

\section{$\underline{\text { Patrón de dominancia plural (frecuencia ordinal) }}$}

1. (258) problemas $\rightarrow$ (280) problema

2. (448) datos $\rightarrow$ (3071) dato

3. (518) dólares $\rightarrow$ (3505) dólar

4. (909) armas $\rightarrow$ (2908) arma

5. (992) flores $\rightarrow$ (2879) flor

6. (1136) alimentos $\rightarrow$ (3265) alimento

7. (1144) brazos $\rightarrow$ (1459) brazo

8. (1529) votos $\rightarrow$ (1868) voto

9. (1531) piernas $\rightarrow$ (3661) pierna

10. (1649) gastos $\rightarrow(2306)$ gasto

11. (1664) negocios $\rightarrow$ (2306) negocio

12. (1723) detalles $\rightarrow$ (2410) detalle

13. (1803) síntomas $\rightarrow$ (6311) síntoma

14. (1865) dedos $\rightarrow$ (3027) dedo

15. (2140) torres $\rightarrow$ (2837) torre

16. (1879) estrellas $\rightarrow$ (2171) estrella

17. (2237) dientes $\rightarrow(9678)$ diente

18. (2572) huevos $\rightarrow$ (3334) huevo

19. (4155) genes $\rightarrow(6672)$ gen

20. (6469) bonos $\rightarrow$ (7026) bono
21. (3762) nubes $\rightarrow(6533)$ nube

22. (4108) rayos $\rightarrow$ (4686) rayo

23. (2427) armadas $\rightarrow$ (2736) armada

24. (2985) huesos $\rightarrow$ (4742) hueso

25. (2375) letras $\rightarrow$ (3048) letra

26. (1147) páginas $\rightarrow(1880)$ página

27. (6265) moléculas $\rightarrow$ (9349) molécula

28. (601) elecciones $\rightarrow$ (1561) elección

29. (263) ojos $\rightarrow$ (1821) ojo

30. (172) personas $\rightarrow(357)$ persona

31. (1754) beneficios $\rightarrow$ (2064) beneficio

32. (1835) células $\rightarrow(5475)$ célula

33. (7709) pulmones $\rightarrow$ (9755) pulmón

34. (7615) mejillas $\rightarrow$ (8934) mejilla

35. (3537) rodillas $\rightarrow(6289)$ rodilla

36. (1848) paredes $\rightarrow(2169)$ pared

37. (1710) labios $\rightarrow(+10000)$ labio

38. (5751) aplausos $\rightarrow(+10000)$ aplauso

39. (2854) lágrimas $\rightarrow(+10000)$ lágrima

40. (3277) zapatos $\rightarrow(+10000)$ zapato 
https://doi.org/10.46744/bapl.201901.008

Aplicando uno de los principios rectores de la lingüística cognitiva, observamos que, en la categoría nocional de dominancia plural, opera la prototipicidad, es decir, hay palabras con dominancia plural difusa y palabras con dominancia plural nítida. En el sector difuso del espectro, tenemos las siguientes palabras:

1. placas (orden 5836) $\rightarrow$ placa (orden 5838)

2. personas (orden 172) $\rightarrow$ persona (orden 357)

3. páginas (orden 1147) $\rightarrow$ página (orden 1880)

4. problemas (orden 258) $\rightarrow$ problema (orden 280)

5. torres (orden 2140) $\rightarrow$ torre (orden 2837)

6. brazos (orden 1144) $\rightarrow$ brazo (orden 1459)

El carácter difuso implica que la diferencia entre las frecuencias normalizadas sea muy pequeña («placas» 15.17 versus «placa»15.16) y que se diluye, en cierto modo, la configuración cognitiva que conlleva la dominancia plural. Así, por ejemplo, la base cognitiva que establece la nítida dominancia plural en el par «piernas» (frecuencia normalizada: 60.86) / "pierna» (frecuencia normalizada: 25.31) se morigera en el par «brazos» (frecuencia normalizada: 79.20) / «brazo» (frecuencia normalizada: 63.82). El sustento para establecer la atenuación y la consiguiente borrosidad de la dominancia tiene que ver con la idea de separación o distancia: las piernas suelen estar más juntas que los brazos. Y esta explicación no es ad hoc porque la noción de separación, incluso, puede cambiar la dominancia: en efecto, la forma singular «oreja» (orden 3183 ) es más frecuente que la forma plural «orejas» (orden 4789).

En el sector nítido del espectro, la dominancia plural prototípica, encontramos las siguientes palabras:
1. datos (orden 448)
$\rightarrow$ dato (orden 3071)
2. dólares (orden 518)
$\rightarrow$ dólar (orden 3505)
3. flores (orden 992)
$\rightarrow$ flor (orden 2879)
4. piernas (orden 1531) $\rightarrow$ pierna (orden 3661)
5. síntomas (orden 1803) $\rightarrow$ síntoma (orden 6311)
6. nubes (orden 3762) $\rightarrow$ nube (orden 6533) 

7. ojos (orden 263)
8. células (orden 1835)
$\rightarrow$ ojo (orden 1821)
9. rodillas (orden 3537)
$\rightarrow$ célula (orden 5475)
10. genes (orden 4155)
$\rightarrow$ rodilla (orden 6289)
$\rightarrow$ gen (6672)

Al encontrar con más facilidad ejemplos prototípicos de dominancia plural (lista en la que podemos incluir a palabras como «labios», «aplausos», «lágrimas» y «zapatos») que ejemplos borrosos o difusos, podemos establecer que la categoría de dominancia plural es un constructo sólido y con valor psicolingüístico. La frecuencia normalizada es un buen indicio de esta nitidez: la forma plural «ojos» tiene un valor de 277.73 y la forma singular «ojo» tiene un valor de 52.50. Sin embargo, esta solidez no entraña que no exista cierta variedad en la esfera semántico-conceptual asociada a la presencia del fonema /s/como marca de plural. En tanto que el polo fonológico puede expresar una gama variable (las diferentes sibilantes, el segmento aspirado y el cero fonético), también postulamos que hay una gama de conceptos asociados con el polo semántico.

Si bien la dominancia plural es una categoría conceptual asociada a la noción de número («más de uno»), consideramos, en virtud de la hipótesis que hemos avanzado, que en el polo semántico podemos encontrar conceptos que brindan prominencia al patrón plural. En efecto, la frecuencia reversible presupone que existen nociones muy fuertes en la mente que determinan la saliencia cognitiva de la pluralidad. Hay, por lo menos, cuatro estratos semánticos asociados con la dominancia plural:

En primer término, una categoría de inherencia plural (sin llegar a la categoría de pluralia tantum) asociada con una pertenencia a una cantidad relativamente grande: datos, dólares, armas, flores, alimentos, votos, gastos, detalles, síntomas, genes, nubes. Aunque no es absurdo pensar estos conceptos en singular (no son, obviamente, pluralia tantum), se trata de entes vinculados con una cantidad mayor.

En segundo término, una categoría de colectividad asociado a una idea de conjunto mayor: estrellas, buevos, torres, armadas, moléculas, paredes. 
Aunque se puede pensar en una estrella singular, un huevo singular, la idea de cohorte tiene más saliencia cognitiva.

En tercer término, una categoría asociada con la intensidad sumativa: beneficios, aplausos, lágrimas, elecciones. Se trata de un plural intensificador que se asocia con una idea de suma, razón por la cual no desaparece la noción de plural. En tal sentido, esta categoría no se identifica con la intensidad sin pluralidad que ha conducido a Concepción Company (2017) a postular la hipótesis de "plurales que no son plurales» y que está en la base de las fórmulas castellanas «buenos días»o «muchas gracias».

En cuarto término, una categoría asociada con la pluralidad experiencial corpórea, de acuerdo con la hipótesis cognitiva de la mente corporizada. Al reconocer la base corpórea de la experiencia humana, nuestra mente reconoce la pluralidad de palabras como piernas, ojos, pulmones, mejillas, rodillas y labios (la noción de dualidad), y de palabras como dedos, dientes y huesos (la noción de colectividad).

\section{Conclusiones}

Respecto de la categoría de dominancia plural, hemos constatado que se da en la lengua española sobre la base de un análisis de una base de datos primordial denominada «Listado de frecuencias» (CREA). La dominancia plural es un caso de frecuencia reversible por cuanto se espera que las formas singulares sean más frecuentes, de acuerdo con el expediente de la ley de Zipf.

En virtud de que el patrón de dominancia plural no se puede dar cuenta con la hipótesis de la economía de los gestos articulatorios, hemos determinado que la dominancia plural se vincula con estratos conceptuales del polo semántico: noción de inherencia plural, noción de colectividad, noción de intensidad sumativa y noción de pluralidad experiencial corpórea. 
https://doi.org/10.46744/bapl.201901.008

\section{BIBLIOGRAFÍA}

ALAMEDA, J. \& CUETOS, F. (1995). Diccionario de frecuencias de las unidades lingüísticas del castellano. Oviedo: Universidad de Oviedo.

ALLEN, L.R. \& GARTON, R.F. (1968). «The influence of wordknowledge on the word-frequency effect in recognition memory». Psychon. Sci., 10(12) pp. 401-402.

BAAYEN, R. H., DIJKSTRA, T. \& SCHREUDER, R. (1997). «Singulars and plurals in Dutch: Evidence for a parallel dual-route model». Journal of Memory and Language, 37, pp. 94-117.

BYBEE, J. (1994). «A view of phonology from a cognitive and functional perspective». Cognitive Linguistics, 5-4, pp. 285-305.

CALUDE, A. \& PAGEL, M. (2011). «How do we use language? Shared patterns in the frequency of word use across 17 languages». Philosophical Transactions of the Royal Society B. 366, pp. 1101-1107.

COMPANY, C. (2017). «Plurales que no son plurales. Una aportación del español a la tipología del reanálisis». Lingüística, 33(1) pp. 73-87.

DEHAENE, S. \& MEHLER, J. (1992). "Cross-linguistic regularities in the frequency of number words». Cognition, 43(1) pp. $1-29$.

DOMÍNGUEZ, A., CUETOS, F. \& SEGUI, J. (1999). «The processing of grammatical gender and number in spanish». Journal of Psycholinguistic Research, 28, pp. 485-498.

KÖHLER, R., ALTMANN, G. \& GRZYBEK, P. (eds.) (2009). Word frequency studies. Berlin: Mouton de Gruyter. 
https://doi.org/10.46744/bapl.201901.008

LEVELT, W. (2001). «Spoken word production: a theory of lexical access». PNAS, November 6, 98(23) pp. 13464-13471.

NEW, B., BRYSBAENT, M., SEGUI, J., FERRAND, L. \& RASTLE, K. (2004). «The processing of singular and plural nouns in French and English». Journal of Memory and Language, 51, pp. 568-585.

NORRIS, D. (2006). «The Bayesian reader: explaining word recognition as an optimal Bayesian process». Psychological Review, 113(2) pp. 327-357.

PINKER, S. (1997). «Words and rules in the human brain». Nature, 387, pp. 547-548.

SERENO, J. \& ALLARD, J. (1997). «Processing of english inflectional morphology». Memory and Cognition, 25, pp. 425-437.

ZIPF, G. (1949). Human behavior and the principle of least effort. New York: Addison-Wesley. 